\title{
BMJ Open Effects of medical and non-medical cannabis use in older adults: protocol for a scoping review
}

Dianna Wolfe, ${ }^{1}$ Kimberly Corace, ${ }^{1,2,3,4}$ Danielle Rice, ${ }^{1,5}$ Andra Smith, ${ }^{6}$ Salmaan Kanji, ${ }^{1,7,8}$ David Conn, ${ }^{9}$ Melanie Willows, ${ }^{3,10,11}$ Gary E Garber, ${ }^{1,12,13}$ John Puxty, ${ }^{14}$ Esther Moghadam, ${ }^{15}$ Becky Skidmore, ${ }^{1}$ Chantelle Garritty, ${ }^{1}$ Kednapa Thavorn, ${ }^{1,13}$ David Moher (D) ,1, 13 Brian Hutton (1) 1,13

To cite: Wolfe D, Corace K, Rice $\mathrm{D}$, et al. Effects of medical and non-medical cannabis use in older adults: protocol for a scoping review. BMJ Open 2020;10:e034301. doi:10.1136/ bmjopen-2019-034301

- Prepublication history and additional material for this paper are available online. To view these files, please visit the journal online (http://dx.doi. org/10.1136/bmjopen-2019034301).

DW and KC contributed equally.

DW and KC are joint first authors.

Received 13 September 2019 Revised 13 January 2020 Accepted 13 February 2020

Check for updates

(c) Author(s) (or their employer(s)) 2020. Re-use permitted under CC BY-NC. No commercial re-use. See rights and permissions. Published by BMJ.

For numbered affiliations see end of article.

Correspondence to

Dr Brian Hutton;

bhutton@ohri.ca

\section{ABSTRACT}

Introduction With its legalisation and regulation in Canada in 2018, the proportion of Canadians reporting cannabis use in 2019 increased substantially over the previous year, with half of new users being aged $45+$ years. While use in older adults has been low historically, as those born in the 1950s and 1960s continue to age, this demographic will progressively have more liberal attitudes, prior cannabis exposure and higher use rates. However, older adults experience slower metabolism, increased likelihood of polypharmacy, cognitive decline and chronic physical/mental health problems. There is a need to enhance knowledge of the effects of cannabis use in older adults. The following question will be addressed using a scoping review approach: what evidence exists regarding beneficial and harmful effects of medical and non-medical cannabis use in adults $>50$ years of age? Given that beneficial and harmful effects of cannabis may be mediated by patient-level (eg, age, sex and race) and cannabis-related factors (eg, natural vs synthetic, consumption method), subgroup effects related to these and additional factors will be explored.

Methods and analysis Methods for scoping reviews outlined by Arksey \& O'Malley and the Joanna Briggs Institute will be used. A librarian designed a systematic search of the literature from database inception to June 2019. Using the OVID platform, Ovid MEDLINE will be searched, including Epub Ahead of Print and In-Process and Other Non-Indexed Citations, Embase Classic+Embase, and PsycINF0 for reviews, randomised trials, non-randomised trials and observational studies of cannabis use. The Cochrane Library on Wiley will also be searched. Eligibility criteria will be older adult participants, currently using cannabis (medical or non-medical), with studies required to report a cannabis-related health outcome to be eligible. Two reviewers will screen citations and full texts, with support from artificial intelligence. Two reviewers will chart data. Tables/graphics will be used to map evidence and identify evidence gaps.

Ethics and dissemination This research will enhance awareness of existing evidence addressing the health effects of medical and non-medical cannabis use in older adults. Findings will be disseminated through a peerreviewed publication, conference presentations and a stakeholder meeting.

Trial registration number DOI 10.17605/OSF.I0/5JTAQ.
Strengths and limitations of this study

- This study will use a rigorous approach to scoping reviews to explore the health effects (both beneficial and harmful) of cannabis in the elderly, addressing a currently important knowledge gap for this population.

- The research will address a large volume of literature which has not previously been synthesised.

- This scoping review will include systematic reviews, randomised and non-randomised studies, and observational data.

- Grey literature will not be reviewed, given the anticipated volume of peer-reviewed literature.

- This review will not formally assess the quality of included studies.

\section{INTRODUCTION}

Until it was legalised in 2018, cannabis was the most widely used illicit substance in Canada. ${ }^{1}$ However, many of the health impacts of cannabis, both positive and negative, have yet to be rigorously studied, given the ethics of conducting randomised controlled trials (RCTs) on illicit substances with perceived harms. Legalisation has increased access to cannabis, resulting in potential benefits as well as potential harms to consumers, including, but not limited to increased risks of substance use disorder, accidents, injuries and presentations to emergency departments. ${ }^{23}$ These potential harms extend across all age groups. However, the effects of the ageing process may mediate many cannabis-related harms in older adults that are not experienced in younger age groups. Although the proportion of middle-aged to older adults reporting cannabis use was relatively low prior to legalisation in October 2018-9\% among those 45 years and older, in early $2018^{4}$-it has risen markedly in the months since legalisation to $14 \%$ in the first quarter of $2019 .{ }^{4}$ In addition 
to legalisation, as the cohort of individuals born in the 1950 s and 1960s ages, it brings with it more liberal attitudes, prior exposure to cannabis and higher use rates. ${ }^{5}$ Despite rising usage rates in this age group, the depth of available evidence regarding the health impacts of cannabis use in older adults is not known.

Cannabinoids are active at the endocannabinoid system (ECS), a series of neuromodulatory lipids and receptors located throughout the central and peripheral nervous systems, immune and hematopoietic systems and many peripheral organs. ${ }^{6}$ The presence of the ECS throughout the body implies the potential for widespread effects of cannabinoids, both beneficial and harmful. Delta-9 tetrahydrocannabinol (THC) and cannabidiol (CBD) are the predominant components of most cannabis products. ${ }^{7}$ As well as some potential therapeutic benefits, THC is responsible for the intoxication and dependence associated with cannabis use and is the primary psychoactive component of natural cannabis. ${ }^{7}$ In contrast, CBD has no intoxicating effects or abuse liability, and because of its widespread activity in the ECS, it has been proposed to be beneficial therapeutically for a variety of health conditions. ${ }^{7}$ Numerous potential therapeutic indications for medical cannabis have been evaluated in the literature, including but not limited to the control of nausea and vomiting associated with chemotherapy, relief of spasticity in multiple sclerosis patients, prevention of graft-versushost disease in allogeneic hematopoietic stem cell transplantation, control of epilepsy and schizophrenia, ocular pressure reduction in glaucoma, HIV/AIDS-associated weight loss, and the control of central, peripheral, and chronic neuropathic pain of various aetiologies. ${ }^{89}$ As with many novel interventions, the results have varied by indication, with demonstrable benefits over harms for only some indications. Cannabis as a medical product became possible with the purification of whole plant extracts from Cannabis sativa L., including purified THC, CBD, and THC and CBD in a 1:1 ratio (nabiximols). ${ }^{8}$ Medical cannabis has been furthered through the development of various synthetic cannabinoids (eg, the synthetic THC analogue, nabilone, and synthetic THC, dronabinol). ${ }^{9}$ Synthetic modification of the molecular structure of THC and CBD to create new synthetic molecules has the potential to widen the range of available cannabis products for medical and non-medical use and their effects on the body.

Generally, older adults suffer from more chronic medical and mental health conditions (eg, chronic pain, insomnia, and mood and cognitive disorders) than younger adults. ${ }^{1011}$ Anecdotal reports suggest that older adults may be attracted to cannabis as a means to ameliorate symptoms of these chronic medical conditions. ${ }^{12}$ As well, lifestyle changes that frequently occur in older adulthood, such as retirement or loss of a spouse, may lead to social isolation, increased leisure time or loss of meaningful work, and contribute to increased cannabis use. ${ }^{12}$ However, while cannabis may be perceived by some patients to improve physical or mental health symptoms, older adults using cannabis either medically or recreationally may be unaware of changes that occur with age that may lead to varying and potentially harmful effects. Past research has demonstrated that cognitive function, attention, memory and executive function-including abilities for impulse control, problem solving and reasoningare reduced with increasing age and that consumption of drugs, including cannabis, has been associated with worsening and/or pronouncement of these deteriorations. ${ }^{13-15}$ Ageing is also associated with structural changes to both grey and white brain matter that correlate with brain function, ${ }^{16}$ and the use of cannabis can exacerbate these structural changes. Polypharmacy of prescription medications is widespread in the older adult population, ${ }^{17}$ and there is some evidence of negative drug interactions between cannabis and prescription and non-prescription medications, ${ }^{18-21}$ another important consideration for older adults. Finally, age-related alterations in the pharmacokinetics of drugs can have a direct impact on the psychoactive effects sought by recreational users, the beneficial health effects sought by medicinal users and the harmful side effects potentially experienced by both. ${ }^{1322}$

Although systematic and scoping reviews have been conducted on cannabis use in younger age groups, ${ }^{23-27}$ age-related changes to the brain and pharmacodynamics suggest that there may be many important differences in the effects of cannabis in older adults compared with younger cohorts. Cannabis research literature is diverse and vast, which challenges systematic review methods. A scoping review would collate and map the available research on cannabis effects in older adults, demonstrating what topic areas may have sufficient evidence for future systematic review. As well, collation and mapping of the research evidence is a first step for the purposes of informing care, developing policy and directing future primary research efforts. A recent overview of systematic reviews evaluating the effectiveness of medical cannabis for any indication identified 73 relevant reviews, ${ }^{28}$ of which 1 was identified as highly relevant to older adults. ${ }^{29}$ In the planned research, we will conduct a scoping review of systematic reviews, RCTs, non-randomised studies (NRS) and observational studies to address the following research questions:

What evidence exists regarding the beneficial and harmful effects of medical and non-medical cannabis use in older adults?

What is known from the existing literature about the beneficial and harmful effects of medical and nonmedical cannabis use in older adults in the following subpopulations, concepts and contexts:

- Age: using older adult age groupings reported in the included literature?

- Sex or gender?

- Race or ethnicity?

- Mental or physical comorbidities?

- Frailty?

- Use of other prescription or non-prescription drugs, alcohol or illicit substances? 
- Consumption method (ie, smoking, vaporising, oils or edibles)?

- Residential setting (eg, community, retirement home or long-term care)?

- Employment status (eg, working or retired) or income level?

- Marital status (eg, single, married, widowed or divorced)?

- Accommodation status (ie, alone, shared or homeless)?

\section{METHODS AND ANALYSIS}

This research will be undertaken using a scoping review approach, underpinned by the framework proposed by Arskey and O'Malley. ${ }^{30} \mathrm{~A}$ scoping review maps the existing sources and types of evidence in a field of interest, and can be used to summarise and disseminate research findings to knowledge users. ${ }^{30}$ Our methods will be guided by several resources, including the scoping review methodology manual published by the Joanna Briggs Institute ${ }^{31}$ and other recent methods guidance. ${ }^{32-34}$

\section{Protocol and registration}

This protocol has been drafted to adhere to the Preferred Reporting Items for Systematic Reviews and Meta-analysis Protocols (online supplementary appendix 1). The protocol has been registered with the Open Science Framework. Given the reflexive and iterative nature of scoping reviews, ${ }^{30}$ amendments to the registered protocol are anticipated and will be described in the final study report.

\section{Eligibility criteria}

Following the guidance of Arskey and O'Malley, our eligibility criteria will be adjusted as we develop familiarity and further expertise with the literature. We based our eligibility criteria on the participants-concept-context criteria $^{31}$ as follows:

\section{Participants}

Adults aged 50 years and older of any sex/gender or race, who currently use cannabis, with or without other substances (eg, tobacco, alcohol or illicit drugs) will be of interest.

\section{Age}

Studies or systematic reviews not explicitly reporting age data but evaluating patients with dementia/Alzheimer's disease, Parkinson's disease, or advanced or end-stage cancer will be included. In a recent review of cannabinoids in palliative medicine, included studies had age ranges of $>50$ years when the population evaluated was patients with Alzheimer's disease or cancer-related pain, anorexia/cachexia, nausea and vomiting, or sleep disturbance. ${ }^{35}$ More conditions specific to older adults may be identified as we progress through the review. Given that many studies will include patients both younger and older than 50 years of age, we will include studies that report age-stratified analyses for an age group of 50 years or older. If age-stratified findings are not reported in a primary study, but $80 \%$ or more of the sample is 50 years of age or older, the study will be included. Similarly, if age data are not reported but patients with any of the health conditions identified previously are included among patients with other health conditions, to be included, the study must have reported a condition-stratified analysis or $80 \%-100 \%$ of the patients must have one of the identified conditions. Therefore, for the purposes of this protocol, 'older adult studies' are those in which (1) $80 \%-100 \%$ of the sample is $50+$ years of age; (2) if age data are not reported, $80 \%-100 \%$ of the sample has dementia/Alzheimer's disease, Parkinson's disease or advanced/end-stage cancer; or (3) an age-stratified or condition-stratified analysis is reported for an age group over 50 years or one of the identified conditions.

\section{Current use}

The definition of 'current use' will likely be variable across studies (eg, daily, weekly, past month or past year); however, we will not include studies evaluating use for more than 1 year in the past. Older adults who are ex-users but are not currently using will not be of interest (eg, those who used as adolescents). Patients with or without a mental or physical health comorbidity will be included. Studies and reviews evaluating younger as well as older adults will be included if data have been reported for an age group of 50 years or older.

\section{Comorbidities}

Examples of comorbidities include cancer (active or in remission), chronic pain, diabetes, anxiety, cognitive decline, dementia, depression, insomnia, post-traumatic stress disorder and schizophrenia.

\section{Concept}

The concept of relevance for the review is characterised further in terms of both the interventions and outcomes of interest for this research, and are as follows.

\section{Interventions}

Medical (ie, either under the care of a medical professional or patient-defined) or non-medical cannabis, of any type, with any mode of consumption (eg, smoking, vaporising oils and edibles), and any dosage will be included. All types of cannabis will be of interest, including whole-plant cannabis; purified whole-plant extracts from C. sativa $\mathrm{L}$. (eg, purified THC, CBD and 1:1 THC:CBD); synthetic cannabinoids, such as synthetic THC (eg, dronabinol and nabilone), CBD and their derivatives, developed through modification of the molecular structure; and other cannabinoids, whether found in the cannabis plant or elsewhere, that are not THC or CBD but that interact with the ECS. ${ }^{36}$

\section{Outcomes}

Both beneficial and harmful effects of cannabis use on physical and mental health will be considered. These will include but will not be limited to the following: 
- Harmful physical health effects (eg, falls, fractures, head injuries, emergency department visits, car accidents, cardiovascular effects, respiratory effects and non-adherence to other drugs).

- Beneficial physical effects (eg, improvements in nausea, vomiting, pain, muscle spasticity, tremors and quality of life).

- Harmful mental health and behavioural outcomes (eg, increased risky, manic and suicidal behaviours; increased cannabis use disorder, cannabis abuse, cannabis dependence or 'problematic' cannabis use; increased or new anxiety, paranoia/psychosis, delirium, depression, sleep disturbance and reduced quality of life).

- Beneficial mental health and behavioural outcomes (eg, decreased risky, manic and suicidal behaviours; decreased cannabis use disorder, cannabis abuse, cannabis dependence, or the word 'problematic' or 'problem' in juxtaposition to the phrase 'cannabis use'; decreased anxiety, paranoia, delirium, depression, chronic pain, sleep, improved quality of life and improved post-traumatic stress disorder).

- Physical brain outcomes (eg, effects on grey matter, white matter integrity, functional connectivity, cortical thickness, total and regional volumes, and surface morphometry/shape).

- Pharmacokinetic impacts (eg, comparative pharmacokinetics of cannabis in older vss younger adults, drug interactions between cannabis and other prescription/non-prescription/illicit drugs).

We will exclude single-arm studies that only report prevalence or incidence of cannabis use.

\section{Context}

Only studies focused on current cannabis consumption will be eligible. All settings are of interest in any geographical area. Consumption of other illicit or prescribed pharmaceuticals will be allowed. All periods of time and duration of follow-up will be eligible.

\section{Types of studies}

Systematic reviews, RCTs, NRSs and observational studies will be included. We will exclude diagnostic test accuracy studies, and studies developing or validating diagnostic criteria for cannabis use disorder or other cannabis-related mental health disorders. Editorials, letters, commentaries, abstracts, case reports and narrative reviews will also be excluded. Only English and French publications will be considered for reasons of timeliness and cost. Grey literature will not be reviewed, given the anticipated volume of peer-reviewed literature to be screened (based on our preliminary search; see online supplementary appendix 2 ), as well as timeline and budget considerations.

We will define a systematic review as being a review with a clearly specified review question that incorporates a systematic search of two or more electronic literature databases, clearly defined eligibility criteria, systematic study selection and data collection by two or more reviewers, an appraisal of the risk of bias of included studies and a synthesis of all information using a quantitative or qualitative approach. Review articles not meeting these criteria will be excluded. NRS may include nonrandomised, quasi-randomised, or single-arm trials (eg, phase I trials). Observational studies of any design will be included, except case reports and case series of fewer than 25 patients. Qualitative studies will be excluded.

\section{Information sources and search strategy}

Preliminary basic searches of the literature identified an extremely high volume of references relevant to medical and non-medical cannabis (eg, >120000 records). We worked closely with an experienced information specialist to iteratively develop a search strategy that will balance the need for inclusivity with the need to yield a citation volume that will be manageable with current reference management software, within the budgetary and time constraints of the review (estimated completion date June 2020). To balance these opposing needs, alternative strategies will be considered, including restriction on date of publication, and application of filters for participant age (ie, $\geq 50$ years of age) or study designs of interest to the identified cannabis literature base.

Using the OVID platform, we will search Ovid MEDLINE, including Epub Ahead of Print and In-Process \& Other Non-Indexed Citations, Embase Classic+Embase and PsycINFO. We will also search the Cochrane Library on Wiley. Databases will be searched from 1947 until 11 June 2019.

Search strategies will use a combination of controlled vocabulary (eg, 'cannabis', 'cannabinoids' and 'marijuana use') and keywords (eg, 'marijuana', 'CBD' and 'Sativex'). Filters for the research designs of interest will be applied to the Ovid searches. Vocabulary and syntax will be adjusted across the databases searched as needed. When possible, animal-only, opinion pieces and case studies will be removed from the search results. Conference abstracts will be removed from Embase and Cochrane CENTRAL. Specific details regarding the strategies are provided in online supplementary appendix 2. The final search strategy will be peer reviewed by another senior information specialist using the Peer Review of Electronic Search Strategies Checklist. ${ }^{37}$

\section{Study selection process and data management}

A sequential approach to study selection will be employed. We will prioritise screening and selection of systematic reviews first, given they are syntheses of findings from primary research studies, followed by NRSs and observational studies, and then RCTs. Non-randomised and observational studies will be prioritised for screening and selection above RCTs due to the expectation that (1) the majority of relevant recreational cannabis research will not be derived from RCTs, given the illegality of recreational cannabis throughout much of the world over the last 20 years; and (2) the expectation that much of the evidence pertaining to applications of medical cannabis 
from RCTs will be identified in included systematic reviews identified earlier in the study selection process. We will iteratively adjust our study selection based on the findings from each search result set, developing stop rules or refining terminology as needed. As noted earlier, any adjustments will be noted in the final study report to maximise transparency in the research approach.

The online systematic review management software DistillerSR will be used for database management and study selection (Evidence Partners, Ottawa, Canada; www. evidencepartners.com). Generally, across the study design strata, two levels of reference screening will be conducted using a priori developed screening forms. A pilot exercise of a random sample of references will be conducted prior to starting each level to ensure high inter-rater reliability. Initially, titles and abstracts will be screened, with those references demonstrating potential relevance progressing to the next level, where their full texts will be assessed for relevance. At both levels, a liberal accelerated approach will be used: one reviewer will be required to include a paper, while agreement of two reviewers will be required to exclude. ${ }^{38}$ Disagreements during title/ abstract screening will result in a reference automatically progressing to the next level, where the full text will provide more information on which to base a decision. At full-text screening, disagreements will be resolved by discussion or by the decision of a third reviewer.

\section{Title/abstract screening}

Initial screening will be designed to rapidly eliminate clearly irrelevant records. For each study design dataset, keyword searches for terms related to adolescents and young adults will be conducted in the titles and abstracts, and the references identified by these searches as related to younger adults/adolescents will be split from the main dataset. Both datasets (ie, the main dataset and the younger adult dataset) will be screened separately using the same methods described further.

Systematic review datasets will be screened with two levels of title/abstract screening: level 1a will screen for terms related to older age and current cannabis use, while level $1 \mathrm{~b}$ will identify references with any cannabisrelated outcomes. Primary study datasets (ie, NRS/observational and RCT) will have a single level of title/abstract screening to identify references of relevance to older adults, current cannabis use and any cannabis-related outcome.

Studies where relevance to older adults is unclear will be included to allow determination of age during fulltext screening (ie, if both younger and older patients are included, the reference will be included at title/abstract screening to determine if disaggregated results were reported in the full text). For title/abstract screening, the terms 'psychedelic' and 'hallucinogen' will be eligible; however, at full-text screening, cannabis use must be explicitly reported. Similarly, for title/abstract screening, any cannabis-related outcome will be eligible, where cannabis is the exposure/intervention (ie, cannabis use should occur prior to the outcome). Case-control studies where a temporal association is not apparent will be included at title/abstract screening for further determination during full-text screening. Cannabis use as an outcome will not be eligible (eg, studies evaluating associations between genes and cannabis use, evaluations of interventions to reduce cannabis use and single-arm studies reporting cannabis prevalence). However, cannabis use disorder (or similar) as an outcome will be eligible, where different types of cannabis use are compared as exposures/interventions. Diagnostic test accuracy evaluations and studies developing or validating diagnostic criteria for cannabis use disorder or other cannabis-related mental health disorders will be excluded.

\section{Full-text screening}

Full-text screening will follow a similar process for all study designs. Initially, references without full texts available in either English or French will be excluded. Subsequently, references that do not report results relevant to older adults will be excluded, followed by those that do not report a relevant cannabis-related outcome and those in which cannabis use is not current. See the 'Eligibility Criteria' section regarding definitions of 'older adult study', 'cannabis-related outcome' and 'current cannabis use'. The following criteria are study-design specific:

- Systematic reviews: must report synthesised results of older adult studies, whether in terms of a metaanalysis or narrative approach. If a narrative summary was used, it must include either quantitative results or a statement of the direction of effect of cannabis use, with or without significance stated. Narrative summaries must appear in the Results section of the review and must not be limited to more general comments within the Discussion section. Reviews that by chance narratively summarise older adult studies, without acknowledging that the patient population is older, will be excluded because the inferences derived from the synthesis by the authors would not have been applied to the context of older adults. For final inclusion, systematic reviews must meet the definition of a systematic review described in the eligibility criteria. Systematic reviews reviewed in full text that reported relevant outcome data for one or more primary studies on older adults among many other primary studies on younger adults will be flagged to capture the citations of the older adult primary studies.

- Primary studies: must meet the definition of 'older adult studies' as defined in the eligibility criteria.

Systematic reviews and primary studies focused strictly on adults over 50 years of age or, if age is not reported, on one of the eligible health conditions will have higher priority for subsequent data charting over studies that also include younger adults or other health conditions.

Use of artificial intelligence (Al) software

Given the large number of anticipated search results, especially for the NRS and observational study stratum 
(>20000 records), we will employ AI methods available in DistillerSR software (Evidence Partners) where deemed feasible and reliable to inform the screening process. The available machine learning engines include both support vector machine (SVM) and naïve Bayes classifiers. We will manually screen through the full text level a set of 300 or more references, which will be used to train the combined SVM and naive Bayes classifiers to generate a probability of relevance score valued at 0 (exclude), 0.5 (unclear) or 1 (include) for each reference in the database. These scores will be used to identify clearly nonrelevant citations (ie, those citations with a probability of 0 ). These citations will be grouped to be checked by a second human reviewer to confirm exclusion. The remaining studies that received probabilities of 0.5 or 1.0 will be sorted according to their relevance probability estimated by the empirical naïve Bayes classifier, which is a continuous score between 0 and 1 , to allow for prioritised screening. The naive Bayes classifier will be rerun and citations reordered after batches of 100 citations or more, depending on the size of the database and the inclusion rate. Prioritised screening will be performed using the liberal accelerated approach described earlier involving two reviewers, with the prioritised element allowing for earlier identification of eligible studies. A flow diagram will be presented in all reports to document the process of study selection.

\section{Data charting}

Included studies will be prioritised for charting by study design. Systematic reviews will be charted first, followed by NRSs and observational studies, then RCTs. RCTs will be charted last, given that most will have already been captured in the data synthesised by the included systematic reviews. Using this approach, if, for example, a large volume of high-quality evidence is identified in systematic reviews related to applications of medical cannabis, it may provide rationale to limit the amount of data extraction from similarly focused RCTs.

A standardised data charting form will be developed in DistillerSR (Evidence Partners, www.evidencepartners. com) that will be refined during the data charting process as reviewers enhance their knowledge of the content area, in keeping with the iterative and reflexive nature of scoping reviews. Prior to data charting from references of a given study design, the charting form will be piloted by all reviewers who will chart data on a random sample of three articles. ${ }^{31}$ Given the large number of anticipated included articles, we will (1) consider charting data in stages, starting with study-level data, then progressing to demographic/context data, then outcomes; and (2) have one reviewer chart study-level and demographic/context data, with a second reviewer verifying this information. To minimise errors of subjective interpretation of information that is critical to the review objectives, charting of the outcomes of each study will be conducted independently by two reviewers followed by conflict resolution by discussion, with input from a third reviewer if necessary. ${ }^{39}$
Items for data charting will include the following information:

- Manuscript/study-level data: study authors; year of publication; country of study or if not reported, country of first author; funding source; study design (ie, systematic review, RCT, NRS and observational study); objective; and sample size. For systematic reviews, the number of included studies and patients will be charted.

- Population demographics: proportion of male/ female/other participants, mean age/age distribution/age-related inclusion criteria, race/ethnicity distribution, employment status distribution, primary residence data (ie, community, retirement home and long-term care facility), marital status data, accommodation status distribution (ie, shared or alone), population data regarding mental health comorbidities (eg, anxiety, depression, insomnia and schizophrenia) and physical health comorbidities (eg, chronic pain, diabetes and cancer), and data regarding couse of other substances (yes/no, specify substances)

- Type of cannabis consumption: medical/nonmedical/mixed, type of cannabis products consumed (eg, whole plant/natural, synthetic and names of strains/synthetic compounds evaluated), mode of consumption (eg, smoking, vaporising, edibles and oils), ratio of THC:CBD, concentration and dose.

- Comparison evaluated: no comparison (ie, useonly single-arm studies) or comparisons of cannabis descriptors (eg, use vs no use, frequencies of use, strain types, THC or CBD concentrations, THC:CBD ratios and modes of consumption) or participant descriptors (eg, sexes/genders, age groups, races/ethnicities, employment statuses, primary residences (ie, community, retirement home and long-term care facility), marital statuses, accommodation statuses (ie, shared or alone), mental health comorbidities, physical health comorbidities and couses of other substances).

- Outcomes: for each outcome of interest reported (see eligibility criteria), the outcome definition, duration of follow-up, direction of effect and significance will be charted. Given this is a scoping review, all outcomes of interest will have equal priority. For systematic reviews, the authors' synthesised findings will be charted.

- Key findings identified by authors that are related to our review objectives.

\section{Critical appraisal of included evidence sources}

Quality appraisal of included systematic reviews will be conducted using the AMSTAR- 2 tool ${ }^{40}$ to identify evidence from high-quality reviews during synthesis. In keeping with scoping review methodology, ${ }^{30} 31$ formal assessment of the risk of bias in primary studies will not be undertaken. 


\section{Synthesis and presentation of the results}

Mapping of the included evidence will be conducted in Microsoft Excel (Office 365), SmartDraw (SmartDraw Software, LLC, San Diego, USA) and other software as needed, with results being presented using a combination of tabular, graphical and narrative approaches. When presenting tabular data, we will group studies based on underlying characteristics of interest, depending on the available data. These characteristics may include study design, analysis type, type of cannabis use (medical vs non-medical) or outcome type reported (ie, mental health/behavioural, physical health, brain and pharmacokinetic). Separate tables will be generated for each study design reviewed (eg, systematic reviews, RCTs, NRSs and observational studies). Organising data by outcome in tables may allow identification of comparisons across study design types while also informing identification of contradictory results, if present. Visualisation of results will be aided by using coloured table cells to indicate presence of subgroups. Similarly, outcome data will be presented with cell colour indicating direction of effect (eg, studies with positive findings for an outcome would receive a green cell, negative findings a red cell and non-significant findings a grey cell). Sample tables have been provided in online supplementary appendix 3. Bar graphs, pie charts, geographical maps, bubble plots and other approaches will also be used to present trends of the evidence base in terms of characteristics, such as year of publication, country of study, patient demographic traits (eg, sex/gender and comorbidities). To augment tabular and graphical presentations, we will also provide structured descriptive summaries of study characteristics and outcomes to elaborate on the evidence base and to identify topics associated with considerable information versus a current lack of primary research. Final reporting of the scoping review will follow the Preferred Reporting Items for Systematic Reviews and Meta-Analyses Extension for Scoping Reviews. ${ }^{41}$

\section{Ethics and dissemination}

Scoping reviews involve the performance of reviewing and collecting data from publicly available information, and thus, this research does not require ethics approval. Strategies for dissemination will include a peer-reviewed publication, conference presentations and engagement with knowledge users as outlined in the Discussion section.

\section{Patient and public involvement}

In planning this research, input was sought from multiple organisations representing individuals with lived experience during the preparation phase regarding elements of its design to ensure its findings would be of relevance to multiple groups, including those with lived experience as well as stakeholders actively engaged in initiatives related to seniors' health. Representatives from these organisations will also be part of a planned stakeholder meeting further described that will inform prioritisation of future research.

\section{DISCUSSION}

\section{Knowledge translation strategies}

Our review will use an integrated knowledge translation approach via the inclusion of our knowledge users (including representation from the Canadian Society of Addiction Medicine, the Canadian Coalition for Seniors' Mental Health, the National Initiative for the Care for the Elderly, the Seniors Health Knowledge Network, the Community Addictions Peer Support Association, Public Health Ontario and Ottawa Public Health) as collaborators throughout the review process. Input on review questions and scope was sought in the design of this protocol to ensure that our work would inform current practice and policy needs. Based on discussion among research team members, a scoping review approach (as opposed to a systematic review) was universally considered most appropriate based on the current uncertainty regarding the availability and nature of evidence of cannabis use specific to the population of older adults. We will continue to consult with our knowledge user collaborators throughout the process of the review on questions of clinical and methodological importance. Manuscripts resulting from the review will be published in open-access journals chosen by the research team. Lay summaries and knowledge mobilisation products for people with lived experience, the community and decision makers will be developed for dissemination on our knowledge users' websites.

\section{Implications}

The findings from this review will form the foundation for a prioritisation exercise with our knowledge users. Shortly after sharing our findings, we will present and discuss them with our knowledge users in a structured webinar. This will be followed by a survey of our knowledge users to establish their perspectives on future research priorities. An online Delphi process will further establish research priorities, as well as the appropriateness of designs for future research (ie, the conduct of de novo primary research to address knowledge gaps versus the performance of full systematic reviews to synthesise evidence, where it already exists).

\section{Potential limitations and mitigation strategies}

This scoping review addresses a very broad topic, and a considerable volume of information is anticipated to be retrieved by our search strategy. Using an unrestricted search strategy would result in a retrieved volume of records that would be unmanageable with current software (ie, $>120000$ references). We will mitigate this challenge in three ways: (1) imposing certain restrictions on the search strategy to reduce to volume of evidence, (2) using AI to aid in screening a large volume of references, and (3) stratifying our approach to screening and 
data charting according to study design, focusing initial intensive efforts on higher levels of evidence. ${ }^{42}$ The use of AI for screening in systematic reviews has become of considerable interest in recent years, ${ }^{43}{ }^{44}$ particularly in the presence of large citation volumes, ${ }^{45}$ and we will employ a conservative approach wherein this tool will not be responsible for any final decisions as to the inclusion status of a study.

Regarding the minimum age criteria to be used for this review ( $50+$ years), this value was selected by the research team following discussions wherein there was a consensus anticipation that there may exist limited data in adults aged $65+$ years. A reduction in the minimum age criteria was considered to allow for a conservative approach to include more data related to the group of older adults.

To increase the transparency of our review methods, we will use the Open Science Framework to record any changes made to our protocol, as anticipated due to the iterative nature of scoping reviews.

Given the expected volume and heterogeneity of the charted evidence, we anticipate potential challenges in determining the most appropriate and useable method of reporting. We will maintain flexibility in the derivation of static tabular and graphical reporting methods while communicating with our knowledge users regarding their needs. Provision of dynamic data options (ie, Excel spreadsheets) will also be considered to allow greater usability of the data.

Recent legalisation of cannabis in several jurisdictions worldwide has made a collation of the available evidence regarding the beneficial and harmful impacts of cannabis use on health imperative. Older adults are a population demonstrating increased levels of cannabis use; however, the natural ageing process may put older adults at risk of adverse health effects from cannabis that may outweigh any benefits realised. The proposed scoping review will map the evidence base specific to older adults to inform decisions related to clinical care, policy and future research directions.

\section{Author affiliations}

${ }^{1}$ Clinical Epidemiology Program, Ottawa Hospital Research Institute, Ottawa, Ontario, Canada

${ }^{2}$ Department of Psychiatry, Faculty of Medicine, University of Ottawa, Ottawa, Ontario, Canada

${ }^{3}$ Institute of Mental Health Research, University of Ottawa, Ottawa, Ontario, Canada

${ }^{4}$ The Royal Ottawa Mental Health Centre, Ottawa, Ontario, Canada

${ }^{5}$ Department of Psychology, McGill University, Montreal, Quebec, Canada

${ }^{6}$ Brain and Mind Research Institute, University of Ottawa, Ottawa, Ontario, Canada

${ }^{7}$ Department of Pharmacy, The Ottawa Hospital, Ottawa, Ontario, Canada

${ }^{8}$ Faculty of Medicine, University of Ottawa, Ottawa, Ontario, Canada

${ }^{9}$ Department of Psychiatry, University of Toronto, Toronto, Ontario, Canada

${ }^{10}$ Substance Use and Concurrent Disorders Program, The Royal Ottawa Mental

Health Centre, Ottawa, Ontario, Canada

${ }^{11}$ Department of Family Medicine, Faculty of Medicine, University of Ottawa, Ottawa, Ontario, Canada

${ }^{12}$ Infection Prevention and Control, Public Health Ontario, Toronto, Ontario, Canada

${ }^{13}$ School of Epidemiology and Public Health, University of Ottawa, Ottawa, Ontario, Canada

${ }^{14}$ Faculty of Medicine, Queen's University, Kingston, Ontario, Canada

${ }^{15}$ Health Promotion, Ottawa Public Health, Ottawa, Ontario, Canada
Twitter Chantelle Garritty @cgarritty and David Moher @dmoher

Acknowledgements We thank Hanan Abramovici for his helpful comments and contributions to the development and review of this protocol.

Contributors $\mathrm{BH}, \mathrm{KC}$ and DW designed the review. DW prepared the first draft of the manuscript. BS created and tested the search strategies to be used in the bibliographic databases. KC, DR, MW and AS provided clinical expertise, and BH and CG provided review expertise during protocol development. All authors provided input in the planning of the study and also reviewed, provided comment and approved the protocol and manuscript. BH conceived of and is the guarantor of the review.

Funding This work was funded as a catalyst grant in 2019 by the Canadian Institutes of Health Research and the Canadian Centre for Substance Use and Addiction.

Disclaimer The funders had no role in the development of the protocol.

Competing interests BH has previously received honoraria from Cornerstone Research Group for methodological advice related to the conduct of systematic reviews and meta-analysis.

Patient consent for publication Not required.

Provenance and peer review Not commissioned; externally peer reviewed.

Open access This is an open access article distributed in accordance with the Creative Commons Attribution Non Commercial (CC BY-NC 4.0) license, which permits others to distribute, remix, adapt, build upon this work non-commercially, and license their derivative works on different terms, provided the original work is properly cited, appropriate credit is given, any changes made indicated, and the use is non-commercial. See: http://creativecommons.org/licenses/by-nc/4.0/.

ORCID iDs

David Moher http://orcid.org/0000-0003-2434-4206

Brian Hutton http://orcid.org/0000-0001-5662-8647

\section{REFERENCES}

1 Rotermann M, Pagé M-M. Prevalence and correlates of non-medical only compared to self-defined medical and non-medical cannabis use, Canada, 2015. Health Rep 2018;29:3-13.

2 Hall W, Lynskey M. Evaluating the public health impacts of legalizing recreational cannabis use in the United States. Addiction 2016;111:1764-73

3 Hajizadeh M. Legalizing and regulating marijuana in Canada: review of potential economic, social, and health impacts. Int $J$ Health Policy Manag 2016;5:453-6.

4 Statistics Canada. National cannabis survey, first quarter 2019. Government of Canada, 2019.

5 Choi NG, DiNitto DM, Marti CN. Older marijuana users: life stressors and perceived social support. Drug Alcohol Depend 2016;169:56-63.

6 Pacher P, Bátkai S, Kunos G. The endocannabinoid system as an emerging target of pharmacotherapy. Pharmacol Rev 2006;58:389-462.

7 Spindle TR, Bonn-Miller MO, Vandrey R. Changing landscape of cannabis: novel products, formulations, and methods of administration. Curr Opin Psychol 2019;30:98-102.

8 Le Boisselier R, Alexandre J, Lelong-Boulouard V, et al. Focus on cannabinoids and synthetic cannabinoids. Clin Pharmacol Ther 2017;101:220-9.

9 Health Canada, Santé Canada. Information for health care professionals: cannabis (marihuana, marijuana) and the cannabinoids : dried or fresh plant and oil administration by ingestion or other means psychoactive agent, 2018.

10 Lyness JM, Caine ED, King DA, et al. Psychiatric disorders in older primary care patients. J Gen Intern Med 1999;14:249-54.

11 Ward BW, Schiller JS. Prevalence of multiple chronic conditions among US adults: estimates from the National health interview survey, 2010. Prev Chronic Dis 2013;10:120203.

12 Dinitto DM, Choi NG. Marijuana use among older adults in the U.S.A.: user characteristics, patterns of use, and implications for intervention. Int Psychogeriatr 2011;23:732-41.

13 Flint AJ, Merali Z, Vaccarino FJ. Improving quality of life: substance use and aging. Ottawa, Ontario: Canadian Centre on Substance Use and Addiction, 2018.

14 Kelleher LM, Stough C, Sergejew AA, et al. The effects of cannabis on information-processing speed. Addict Behav 2004;29:1213-9. 
15 Ranganathan M, D'Souza DC. The acute effects of cannabinoids on memory in humans: a review. Psychopharmacology 2006;188:425-44.

16 Kaag AM, Schulte MHJ, Jansen JM, et al. The relation between gray matter volume and the use of alcohol, tobacco, cocaine and cannabis in male polysubstance users. Drug Alcohol Depend 2018;187:186-94.

17 Rotermann M, Sanmartin C, Hennessy D, et al. Prescription medication use by Canadians aged 6 to 79. Health Rep 2014;25:9.

18 Yamreudeewong W, Wong HK, Brausch LM, et al. Probable interaction between warfarin and marijuana smoking. Ann Pharmacother 2009;43:1347-53.

19 McLeod AL, McKenna CJ, Northridge DB. Myocardial infarction following the combined recreational use of Viagra and cannabis. Clin Cardiol 2002;25:133-4.

20 Wilens TE, Biederman J, Spencer TJ. Case study: adverse effects of smoking marijuana while receiving tricyclic antidepressants. J Am Acad Child Adolesc Psychiatry 1997;36:45-8.

21 Kosel BW, Aweeka FT, Benowitz NL, et al. The effects of cannabinoids on the pharmacokinetics of indinavir and nelfinavir. AIDS 2002;16:543-50.

22 Mangoni AA, Jackson SHD. Age-related changes in pharmacokinetics and pharmacodynamics: basic principles and practical applications. Br J Clin Pharmacol 2004;57:6-14.

23 Aviram J, Samuelly-Leichtag G. Efficacy of cannabis-based medicines for pain management: a systematic review and meta-analysis of randomized controlled trials. Pain Physician 2017;20:E755-96.

$24 \mathrm{Lim} \mathrm{K}$, See YM, Lee J. A systematic review of the effectiveness of medical cannabis for psychiatric, movement and neurodegenerative disorders. Clin Psychopharmacol Neurosci 2017;15:301-12.

25 Jouanjus E, Raymond V, Lapeyre-Mestre M, et al. What is the current knowledge about the cardiovascular risk for users of cannabis-based products? A systematic review. Curr Atheroscler Rep 2017:19:26.

26 Nugent SM, Morasco BJ, O'Neil ME, et al. The effects of cannabis among adults with chronic pain and an overview of general harms: a systematic review. Ann Intern Med 2017;167:319-31.

27 National Academies of Sciences, Engineering, and Medicine. The health effects of cannabis and cannabinoids: the current state of evidence and recommendations for research. Washington, DC: The National Academies Press, 2017.

28 Pratt M, Stevens A, Thuku M, et al. Benefits and harms of medical cannabis: a scoping review of systematic reviews. Syst Rev 2019;8:320.

29 van den Elsen GAH, Ahmed AlA, Lammers M, et al. Efficacy and safety of medical cannabinoids in older subjects: a systematic review. Ageing Res Rev 2014;14:56-64.
30 Arksey H, O'Malley L. Scoping studies: towards a methodological framework. Int J Soc Res Methodol 2005;8:19-32.

31 Peters MDJ, Godfrey C, Mclnerney P. Chapter 11: Scoping reviews. In: Aromataris E, Munn Z, eds. Joanna Briggs Institute reviewer's Manual, 2017. https://reviewersmanual.joannabriggs.org/

32 Levac D, Colquhoun H, O'Brien KK. Scoping studies: advancing the methodology. Implement Sci 2010;5:69.

33 Peters MDJ, Godfrey CM, Khalil H, et al. Guidance for conducting systematic scoping reviews. Int J Evid Based Healthc 2015;13:141-6.

34 Thomas A, Lubarsky S, Durning SJ, et al. Knowledge syntheses in medical education: Demystifying scoping reviews. Acad Med 2017;92:161-6

35 Mücke M, Weier M, Carter C, et al. Systematic review and metaanalysis of cannabinoids in palliative medicine. $J$ Cachexia Sarcopenia Muscle 2018;9:220-34.

36 Morales P, Reggio PH, Jagerovic N. An overview on medicinal chemistry of synthetic and natural derivatives of cannabidiol. Front Pharmacol 2017;8:422.

37 McGowan J, Sampson M, Salzwedel DM, et al. PRESS Peer Review of Electronic Search Strategies: 2015 Guideline Statement. J Clin Epidemiol 2016;75:40-6.

38 O'Blenis P. One simple way to speed up your screening process, 2017. Available: https://blog.evidencepartners.com/one-simple-wayto-speed-up-your-screening-process [Accessed 21 May 2019].

39 Higgins JPT, Green S, eds. Cochrane Handbook for Systematic Reviews of Interventions Version 5.1.0 [updated March 2011], 2011.

40 Shea BJ, Reeves BC, Wells G, et al. AMSTAR 2: a critical appraisal tool for systematic reviews that include randomised or nonrandomised studies of healthcare interventions, or both. $B M J$ 2017;358:j4008.

41 Tricco AC, Lillie E, Zarin W, et al. PRISMA extension for scoping reviews (PRISMA-ScR): checklist and explanation. Ann Intern Med 2018;169:467.

42 CEBM. Oxford centre for evidence-based medicine - levels of evidence (March 2009), 2009. Available: https://www.cebm.net/2009/ 06/oxford-centre-evidence-based-medicine-levels-evidence-march2009/ [Accessed 21 May 2019].

43 Marshall IJ, Wallace BC. Toward systematic review automation: a practical guide to using machine learning tools in research synthesis. Syst Rev 2019;8:163.

44 Wallace BC, Dahabreh IJ, Schmid CH, et al. Modernizing the systematic review process to inform comparative effectiveness: tools and methods. J Comp Eff Res 2013;2:273-82.

45 Shemilt I, Simon A, Hollands GJ, et al. Pinpointing needles in giant haystacks: use of text mining to reduce impractical screening workload in extremely large scoping reviews. Res Synth Methods 2014;5:31-49. 